\title{
Self-assembly Routes towards Creating Superconducting and Magnetic Arrays
}

\author{
A.A.Zhukov, E.T.Filby, A.V.Goncharov, M.A.Ghanem ${ }^{a}$, \\ P.N.Bartlett ${ }^{a}$, R. Boardman ${ }^{b}$, H.Fangohr ${ }^{b}$, \\ V.V.Metlushko ${ }^{c}$, V. Novosad ${ }^{d}$, G. Karapetrov ${ }^{d}$ \\ and P. A. J. de Groot
School of Physics and Astronomy, University of Southampton, Southampton, SO17 1BJ, UK
${ }^{a}$ School of Chemistry, University of Southampton, Southampton, SO17 1BJ, UK
${ }^{b}$ School of Engineering Sciences, University of Southampton, Southampton, SO17 1BJ, UK
${ }^{c}$ Department of Electrical and Computer Engineering, University of Illinois at Chicago, Chicago, IL 60607-0024, USA
${ }^{d}$ Materials Science Division, Argonne National Laboratory, 9700 South Cass Ave., Argonne, IL60439, USA

Using self-assembly from colloidal suspensions of polystyrene latex spheres we prepared well-ordered templates. By electrochemical deposition of magnetic and superconducting metals in the pores of such templates highly ordered magnetic and superconducting anti-dot nano-structures with $3 D$ architectures were created. Further developments of this template preparation method allow us to obtain dot arrays and even more complicated structures. In magnetic anti-dot arrays we observe a large increase in coercive field produced by nanoscale (50-1000nm) holes. We also find the coercive field to demonstrate an oscillatory dependence on film thickness. In magnetic dot arrays we have explored the genesis of $3 D$ magnetic vortices and determined the critical dot size. Superconducting $\mathrm{Pb}$ anti-dot arrays show pronounced Little-Parks oscillations in $T_{c}$ and matching effects in magnetization and magnetic susceptibility. The spherical shape of the holes results in significantly reduced pinning strength as compared to standard lithographic samples. Our results demonstrate that self-assembly template methods are emerging as a viable, low cost route to prepare sub-micron structures.

PACS numbers: $74.25 \mathrm{Ha}, 75.75+a$. 


\section{A.A.Zhukov et al.}

\section{INTRODUCTION}

Nanostructured materials open new horizons in the development of Solid State physics. A large impact is expected for industrial applications of nanostructured objects. However, the old top-down approach has nearly exhausted its potential. Smaller scales can be reached using electron or ion beam based techniques but they are slow and very expensive. The main requirement for commercially viable products is an efficient and low-cost preparation process. Methods based on templates formed by the self-assembly of colloidal particles have demonstrated promise for a number of applications such as photonic materials ${ }^{1,2}$, microchip reactors ${ }^{3}$ and biosensors ${ }^{4}$. We use ordered templates for the electro-deposition of different magnetic and superconducting materials. This technique offers new opportunities, which are not easily realized by standard lithographic methods, and allows us to create magnetic nano-structures with 3D architectures on a broad range of length scales, 20 - $2000 \mathrm{~nm}$. In this paper we briefly review our previous results ${ }^{5-13}$ and in more detail describe some recent observations. We show that the $3 \mathrm{D}$ architecture of these structures produces many unique properties absent in nanostructures prepared by conventional lithographical techniques.

\section{PREPARATION AND MEASUREMENT TECHNIQUES}

Using slow evaporation of a colloidal water suspension containing 1wt.\% of latex spheres, well-ordered templates have been self-assembled on glass substrates with two sputterd buffer layers: the first Cr (10nm) and the second $\mathrm{Au}$ or $\mathrm{Cu}(200 \mathrm{~nm})$. These templates have been used as moulds to prepare nano-porous magnetic and superconducting structures by electrodeposition methods ${ }^{7}$. After deposition the latex spheres can be removed by dissolving in toluene. Using this method, well-ordered nano-structured 3D arrays were prepared for various magnetic materials such as cobalt, iron, nickel and soft-magnetic $\mathrm{Ni}_{50} \mathrm{Fe}_{50}$ alloy. Lead was used for the preparation of superconducting arrays. A scanning electron microscope (SEM) image of one of the nano-structured films is given in Fig. 1a. It shows that the films have excellent hexagonal order. In comparison with conventional lithographical techniques our method has a significant advantage in that it also produces structuring in the direction transverse to the plane of the film. Cross-sectional SEM (Fig. 1b) demonstrates that the transverse structuring is well ordered too.

We have also developed a new technique ${ }^{8,9}$ to prepare highly-regular arrays of submicron dots, which is called a double templated electrodeposition (DTE) method. Using this DTE method we fabricated highly ordered 


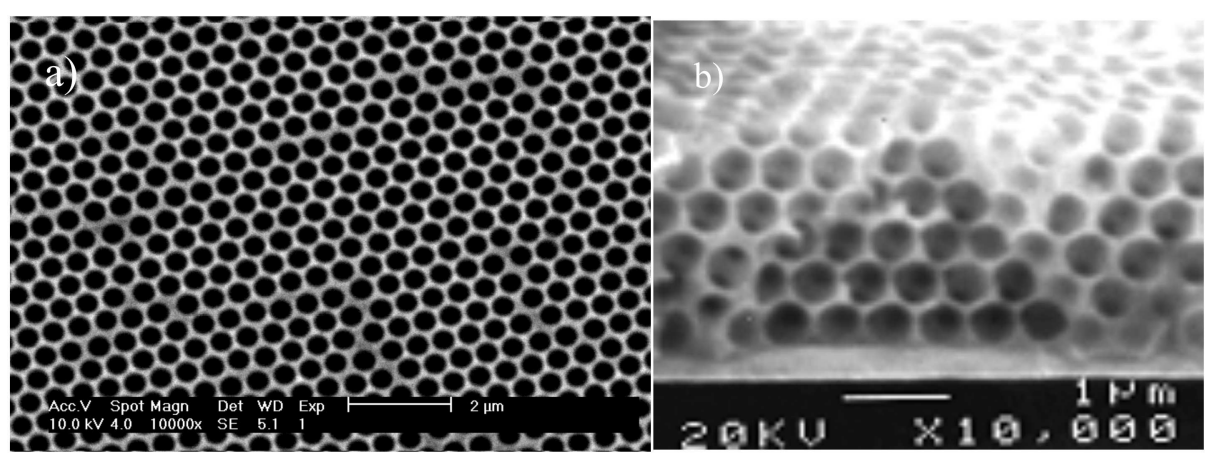

Fig. 1. SEM image of a Ni film with anti-dots prepared using $500 \mathrm{~nm}$ spheres (a) and transverse view of a film after cleaving (b).

arrays of isolated or interconnected magnetic dots with variable diameters (from $20 \mathrm{~nm}$ to half the array period) and periods ranging from 100 to 700 $\mathrm{nm}$. These samples were produced by electrochemical deposition of magnetic metals inside the holes of macroporous poly(pyrrole) polymer templates, which were initially prepared by electrodeposition using primary templates from polystyrene spheres self-assembled on Au substrate. Both Ni and $\mathrm{Co}$ dot arrays have been deposited. Fig. 2 presents an SEM image for a Ni dot array prepared using the DTE method. The nano-crystal structure, composition and morphology of the films were characterised using SEM, energy dispersive X-ray spectroscopy (EDSRX) and X-ray diffraction.

Magnetic measurements were performed using a vibrating sample magnetometer and magneto-optical Kerr effect (MOKE) rig. Properties of magnetic nanostructures were measured at room temperature with the external magnetic field applied parallel to the plane of the film. For superconducting samples a transverse magnetic field direction was used.

\section{MEASUREMENT RESULTS AND DISCUSSION}

Three different types of samples have been studied. Most measurements were carried out for periodic magnetic anti-dot and dot arrays, which will be discussed in sections 3.1 and 3.2, respectively. Results for the superconducting anti-dot structures will be presented in section 3.3. In this paper we focus on specific features in the behaviour of our structures, which result from the 3D architecture and spherical shape of the holes or dots. 


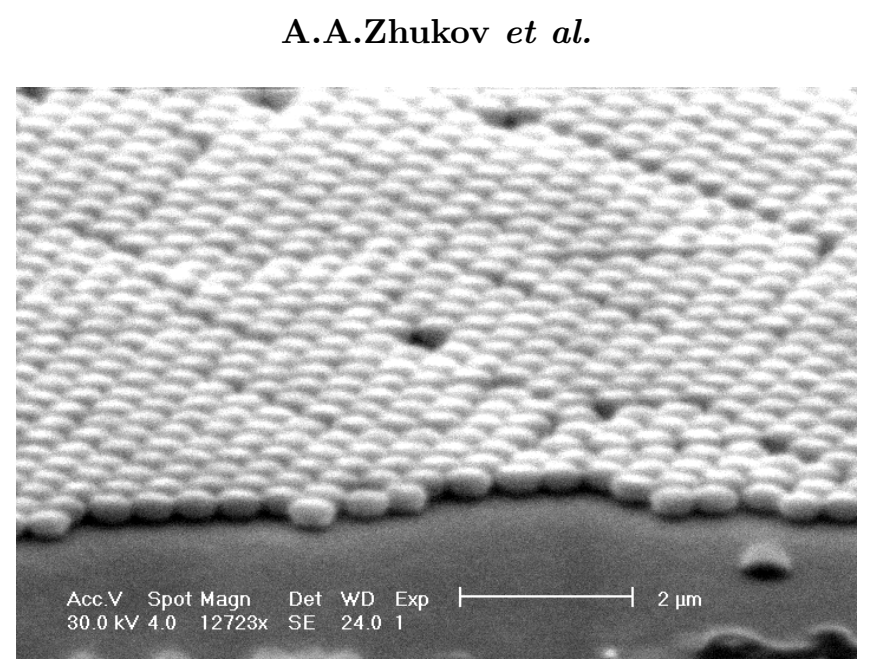

Fig. 2. SEM image of a dot array prepared using 500nm spheres.

\subsection{Magnetic Anti-dots}

In magnetic anti-dot samples nano-structuring significantly changes magnetization loops and value of the coercive field as we have described previously ${ }^{5,6}$. The coercive field, $B_{c}$, shows a non-monotonic change with variation of the diameter $d$ of spherical holes. When pore size decreases $(1000 \mathrm{~nm} \geq d \geq 50$ $\mathrm{nm})$, the coercivity increases by more than an order of magnitude at first, but then, after reaching a maximum, $B_{c}$ starts to decrease. Our $B_{c}(d)$ data resembles that of disordered particulate magnetic materials ${ }^{14,15}$. However, in contrast to our samples, particulate materials correspond to dot, rather than anti-dot, structures. We found the value of the diameter corresponding to maximum coercivity, $d_{\max }$ to agree with the condition $d_{\max } / 2 \approx w_{B}$, where $w_{B}$ is the magnetic domain wall width for the material ${ }^{6}$.

Measurements of the dependence of $B_{c}$ on the thickness $t_{f}$ of the magnetic film revealed a novel effect ${ }^{10}$. Although these films have a homogeneous composition of magnetic material, as evident from the EDSRX studies, we have found that the coercive force changes periodically with film thickness. This is a clear manifestation of the periodical patterning in the direction transverse to the film plane and the 3D architecture of these structures. Fig. 3 demonstrates that $B_{c}$ shows clear oscillations and reaches a maximum for the case when the top surface of the film is near the center of a layer of close packed spherical voids. For complete spherical layers the coercive field approaches a minimum. These observations suggest that the points where the spheres touch play an important role in domain wall pinning and hence the coercivity. 


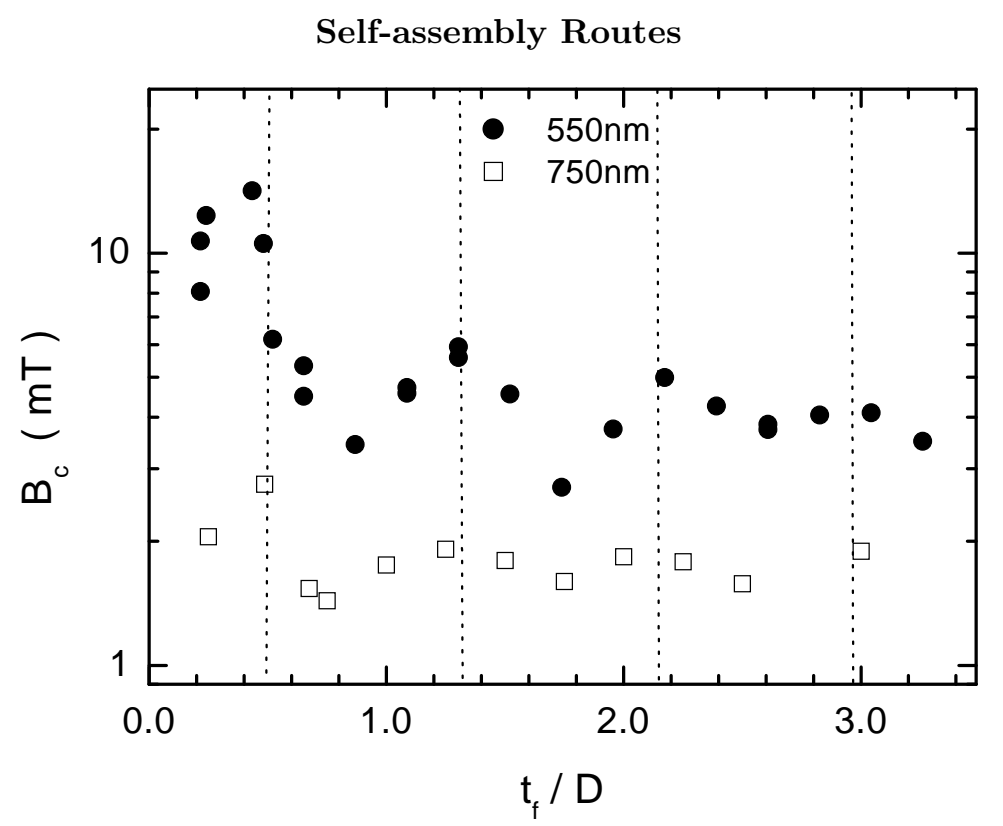

Fig. 3. Coercive field for $\mathrm{Ni}_{50} \mathrm{Fe}_{50}$ films with different thickness prepared using $550 \mathrm{~nm}$ and $750 \mathrm{~nm}$ spheres. For clarity, in the case of $750 \mathrm{~nm}$ the $B_{c}$ values are divided by factor 2 . The dashed lines show the positions of sphere centers for each layer in a close packed structure.

We can qualitatively understand the results of our experiment assuming that domain wall pinning is the main mechanism for the coercive properties. In this case the narrow constrictions between the spherical voids are dominant pinning sites. The number of effective pinning sites changes periodically with the film thickness $t_{f}$. The experimetal behaviour was reproduced using both a flat domain wall pinning model and 2D Monte-Carlo simulations of a multilayer model of our 3D structures ${ }^{10}$.

\subsection{Magnetic Dots}

Previously we have shown that the magnetization curves for quasispherical (spheroidal) dots are significantly different from the thin discs resulting from lithography ${ }^{9,11}$. In the latter case the magnetization curve $M(B)$ has three different regimes (e.g., Ref. 16). They correspond to two oppositely directed saturated states and an intermediate vortex state. The direction of the vortex core is always transverse to the disc plane. As can be seen from Fig. 4 the transitions between these states are sharp and the magnetization curve demonstrates siginificant irreversibility. In contrast to 


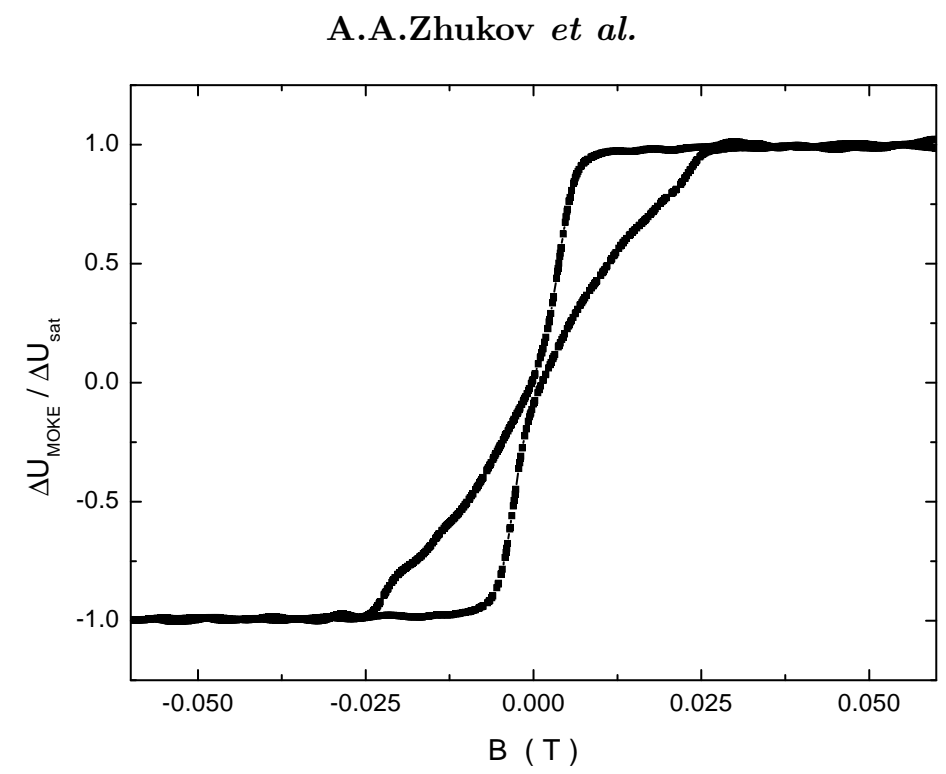

Fig. 4. Magnetization loop for a square array of permalloy dots with diameter $D=1000 \mathrm{~nm}$, period $d=1500 \mathrm{~nm}$ and thickness $t_{f}=15 \mathrm{~nm}$, prepared by e-beam lithography ${ }^{17}$

this, for spheroidal dots we observe four different regimes.

In Fig. 5a the experimental magnetization curves obtained by MOKE for a Ni dot array prepared using 500nm spheres are shown. Numerical simulations using the OOMMF software package ${ }^{18}$ reproduce our experimental results well, Fig. 5b. According to these simulations, in spheroidal dots the saturated state first transforms into a vortex state with the vorticity vector $\nabla \times \mathbf{M}$ directed along the applied magnetic field. Only at smaller fields is this longitudinal vortex replaced by a conventional transverse vortex. Finally for large negative magnetic fields, the fourth regime corresponding to the saturated state with the opposite magnetic moment direction is realised. In spheroidal dots transitions between different regimes are quite smooth resulting in nearly reversible magnetic behaviour. Yet it is worth noting that the experiment shows slightly sharper transition between the vortex and saturated states.

For the description of magnetic vortex behaviour in our experiments we will use the approximation of a coreless vortex, which is similar to the London approximation in superconductors ${ }^{19}$. We expect this model to be applicable for sufficiently large dots. It is too simplified to obtain quantitative agreement with the experiment. However, as we will see, it qualitatively explains our experimental results and is consistent with the numerical simulations ${ }^{11}$. The energy of the coreless vortex is determined by the exchange energy 


\section{Self-assembly Routes}

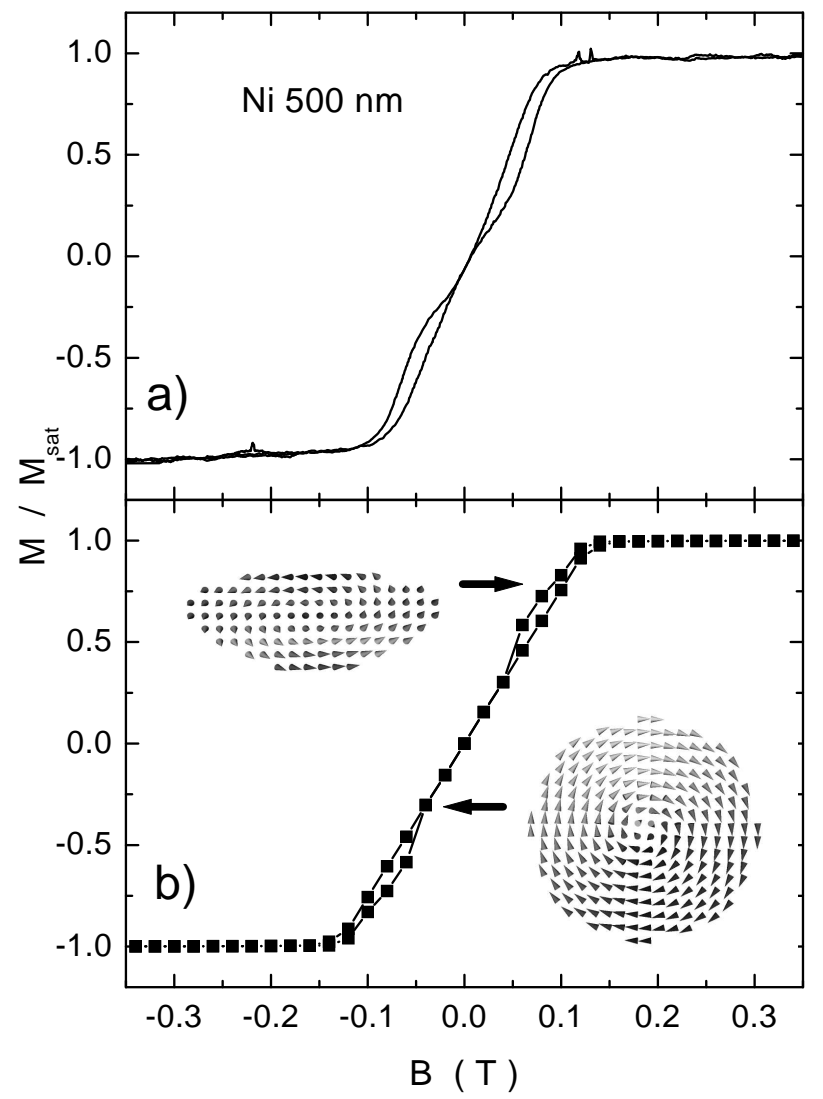

Fig. 5. Magnetization loops for Ni spheroidal dot array with period $500 \mathrm{~nm}$ and thickness $\sim 200 \mathrm{~nm}$ from the experiment (a) and numerical simulations(b). The insets show longitudinal (left) and transverse (right) vortex states

$E_{e x}=A \int\left(\nabla \frac{\mathrm{M}}{M_{S}}\right)^{2} d^{3} \mathbf{r}$. We will use the exchange parameter $A=3.4 \mathrm{pJ} / \mathrm{m}$ and the saturation magnetization $M_{S}=0.493 \mathrm{MA} / \mathrm{m}$ as known for $\mathrm{Ni}^{20}$. For cylindrical symmetry we have $\left(\nabla \frac{\mathrm{M}}{M_{S}}\right)^{2}=\frac{A}{\rho^{2}}$, where $\rho$ is the distance to the rotation axis ${ }^{21}$. Integrating this for a disc and an ellipsoid we get the same value within logarithmic accuracy $E_{e x}=2 \pi L A \ln \left(\frac{R}{a}\right)$. Here $L$ and $R$ are length and external radius of the vortex, and we use the crystal period $a$ as a lower integration limit. Therefore, the dependence on radius is weak and at remanence the shorter transverse vortex has smaller energy. Hence except for the case of a nearly full sphere the tranverse vortex is realised for $B=0$. However, with increasing magnetic field the energy of a longitudinal 


\section{A.A.Zhukov et al.}

vortex drops fast and it becomes energetically more favorable ${ }^{22}$. For $B=0$, comparing the coreless vortex energy with the demagnetizing energy of the monodomain state $E_{d m}=N \mu_{o} M_{S}^{2} V / 2(N$ and $V$ are the demagnetizing factor and volume of a dot), we can find two values for the critical radius for the transition of a spheroidal dot (with $L / R=6 / 7$ similar to our experiment) in a monodomain state. For in-plane magnetic fields we find $R_{c}^{/ /}=$ $71 \mathrm{~nm}$, and $R_{c}^{\perp}=26 \mathrm{~nm}$ for out-of-plane B. These values are in reasonable agreement with results of experiments, $R_{c}^{/ /} \approx 100 \mathrm{~nm}^{12}$, and of numerical simulations, $R_{c}^{/ /}=65 \mathrm{~nm}$ and $R_{c}^{\perp}=45 \mathrm{~nm}$.

\subsection{Superconducting Nanostructures}

The nanostructured $\mathrm{Pb}$ films demonstrate a sharp superconducting transition close to the transition point of $7.18 \mathrm{~K}$ for bulk material ${ }^{23}$. The superconducing transition temperature shows pronounced oscillations with the magnetic field. Commensurability results in sharp peaks at $H_{n}=n \Phi_{o} / S$ with $n=0, \pm 1, \pm 2, \ldots$ Here $\Phi_{o}$ is the flux quantum and $S=3^{1 / 2} d^{2} / 2$ ( $d$ is the period of the hexagonal structure). The peaks broaden with $n$. The amplitude of oscillations exceeds $1 \%$ of $T_{c}$, which is comparable to results for lithographically prepared structures ${ }^{24}$. As can be seen from Fig. 6a the acsusceptibility measurements also reveal commensurability oscillations in the pinning strength ${ }^{13}$. In a wide temperature range sharp peaks are observed near the commensurate points. This tooth-like behaviour was present in all studied samples, with a range of periods $(500 \mathrm{~nm} \leq d \leq 2000 \mathrm{~nm})$, and is different from plain lithographic structures. As shown in Fig. 6b for lithographic samples the dominant feature is a step-like change in pinning strength at commensurate fields. We should also stress that the hole configuration does not affect this difference. Lithographic samples with hexagonal arrays of holes also demonstrate step-like behaviour ${ }^{25}$.

We relate these differences in commensurability behaviour to the spherical shape of the pores in our array. Their smoothly varying pinning potential results in significantly smaller critical currents than in the case of sharp (steep edged) lithographic structures. In the first approximation the difference in the critical currents is governed by the ratio of pinning potential range, which correspond to the radius of the spherical cavity $R$ in our case and the coherence length $\xi$ in sharp lithographic structures ${ }^{26}$. From the measurements of $B_{c 2}$ we find for our sample $\xi(0)=20 \mathrm{~nm}$. In accordance with this the critical currents in the sample with spherical holes $\left(j_{c}\left(T=0.94 T_{c}\right) \approx 4 \cdot 10^{3} \mathrm{~A} / \mathrm{cm}^{2}\right.$ and $\left.j_{c}\left(T=0.87 T_{c}\right) \approx 8 \cdot 10^{4} \mathrm{~A} / \mathrm{cm}^{2}\right)$ are significantly lower than in the film with lithographically prepared disc- 


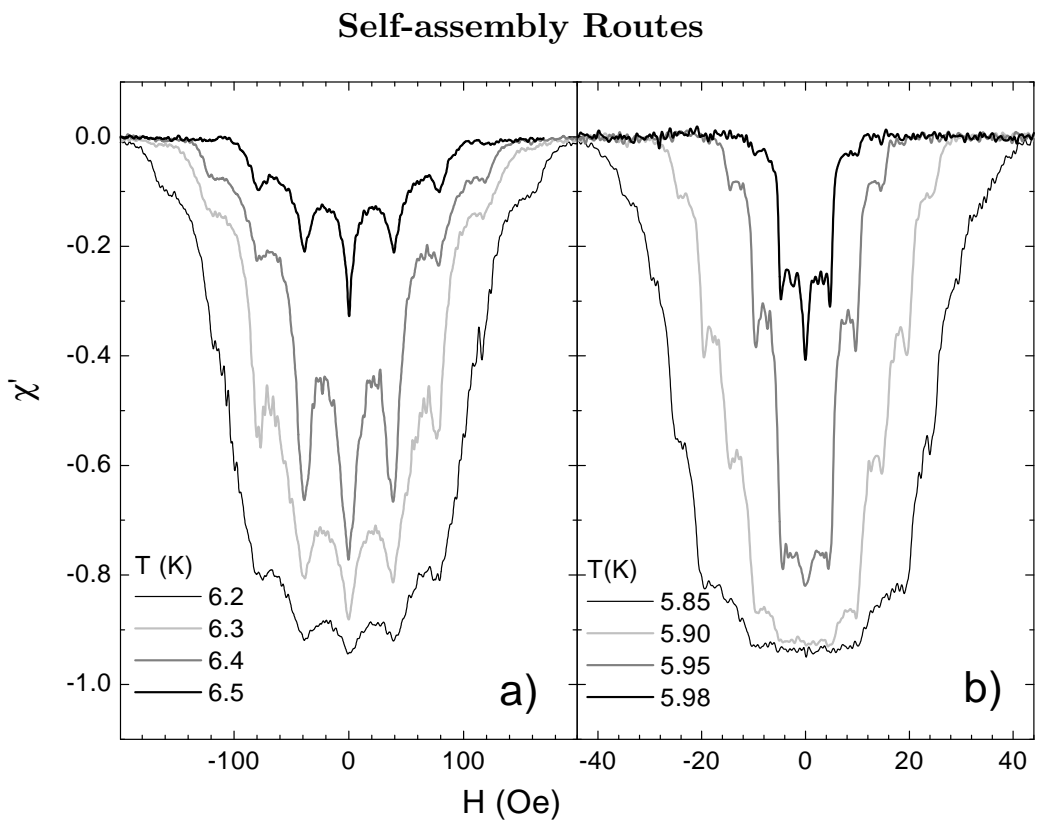

Fig. 6. Field dependence of ac-susceptibility in $\mathrm{Pb}$ films with spherical (a) and cylindrical holes (b). Array parameters: (a) $d=700 \mathrm{~nm}, t_{f}=350 \mathrm{~nm}$ $T_{c}=6.55 \mathrm{~K}$ and $(\mathrm{b}) d=2000 \mathrm{~nm}, D=1000 \mathrm{~nm}, t_{f}=60 \mathrm{~nm}, T_{c}=6.05 \mathrm{~K}$

shape holes ${ }^{25}\left(j_{c}\left(T=0.94 T_{c}\right) \approx 1.7 \cdot 10^{5} \mathrm{~A} / \mathrm{cm}^{2}\right.$ and $j_{c}\left(T=0.87 T_{c}\right) \approx$ $\left.9 \cdot 10^{5} \mathrm{~A} / \mathrm{cm}^{2}\right)$.

Critical currents are determined by the interplay of pinning, $E_{p}$, and vortex lattice deformations, $E_{\text {def }}$, energies. In the general case this involves a very complicated force summation problem. However, the situation simplifies for two extreme cases. For $E_{p} \gg E_{\text {def }}$ we have to deal with the case of strong pinning, which can be adequately described by a single vortex approximation. In this case we expect steps in the pinning force at commensurate points because the pinning force $f_{p}$ decreases with the number of vortices $\mathrm{N}$ pinned on the hole. According to Ref. 27 in long cylindrical holes it follows $f_{p} \propto(1-2 N \xi / R)$. This case corresponds to sharp lithographic structures. In the opposite case of $E_{p} \ll E_{\text {def }}$ we have weak pinning. Here a rigid lattice is a good approximation. The resulting pinning force should then have a maximum at commensurate states and vanish with discommensuration. Such a situation corresponds to our samples with spherical holes. In the lithographic samples in close vicinity of $T_{c}$ the thermal depinning strongly suppresses the pinning force ${ }^{28}$ and we should observe a transition from steps to peaks at the commensurate states. 


\section{A.A.Zhukov et al.}

\section{CONCLUSIONS}

Our results demonstrate that self-assembly template electrodeposition methods offer a very efficient route to create nanostructures on scales from $20 \mathrm{~nm}$ to several micrometers. The prepared anti-dot structures with spherical pores have pronounced 3D architecture. Spheroidal dots can be realised either in connected or disconnected periodical arrays.

The prepared structures reveal unique properties absent in nanostructures prepared by conventional lithographical techniques. In particular, the coercive field of antidot arrays was found to demonstrate a novel oscillatory dependence on film thickness. The observed coercivity data demonstrates that 3D patterned magnetic materials are prototypes of a new class of geometrical multilayer structures in which the layering is due to local shape effects rather then compositional differences. In magnetic dot arrays we have explored the nature of 3D magnetic vortices. In this case, in contrast to standard disc-shaped dots, the direction of the magnetic vortices is not locked to the normal of the dot. The suppression of shape induced magnetic anisotropy in our spheroidal dots is an important factor for the new behaviour of magnetic vortices. In superconducting anti-dot arrays the spherical shape of the holes results in significantly reduced pinning strength and in commensurability behaviour different from standard lithographically prepared arrays.

\section{ACKNOWLEDGMENTS}

This research has been supported by EPSRC grants GR/S13200/01 and GR/S49162/01.

\section{REFERENCES}

1. Y.A. Vlasov, X.-Z. Bo, J.C. Sturm and D.J. Norris, Nature 414, 289 (2001).

2. V.N. Bogomolov, S.V. Gaponenko, I.N. Germanenko, A.M. Kapitonov, E.P. Petrov, N.V. Gaponenko, A.V. Prokofiev, A.N. Ponyavina, N.I. Silvanovich and S.M. Samoilovich, Phys. Rev.E 55, 7619 (1997).

3. H. Gau, S. Herminghaus, P. Lenz, and R. Lipowsky, Science 283, 46 (1999).

4. O.D. Velev and E.W. Kaler, Langmuir 15, 3693 (1999).

5. A.A. Zhukov, A.V. Goncharov, P.A.J. de Groot, P.N. Bartlett, M.A. Ghanem, H. Kupfer, R.J. Pugh, G.J. Tomka, IEE Proc.-Sci.Meas.Technol., 150, 257 (2003).

6. A.A. Zhukov, A.V. Goncharov, P.A.J. de Groot, P.N. Bartlett and M.A. Ghanem. J.Appl.Phys., 93, 7322 (2003). A.A. Zhukov, M.A. Ghanem, A.V. 


\section{Self-assembly Routes}

Goncharov, P.A.J. de Groot, I.S. El-Hallag, P.N. Bartlett, R. Boardman and H. Fangohr, JMMM 272-76, 1621 (2004).

7. P.N. Bartlett, M.A. Ghanem, I.S.El Hallag, P. de Groot and A. Zhukov, J.Mater.Chem. 13, 2596 (2003).

8. M.A. Ghanem , P.N. Bartlett, P. de Groot and A. Zhukov, Electrochemistry Communications, 6, 447 (2004).

9. A.A. Zhukov, M.A. Ghanem, A.V. Goncharov, P.N. Bartlett and P.A.J. de Groot, JMMM 272-76, E1369 (2004).

10. A.A. Zhukov, M.A. Ghanem, A.V. Goncharov, R. Boardman, V. Novosad, G. Karapetrov, H. Fangohr, P.N. Bartlett and P.A.J. de Groot, \# condmat/0406091.

11. R.P. Boardman, H. Fangohr, S.J. Cox, A.V. Goncharov, A.A. Zhukov, P.A.J de Groot, J.Appl.Phys., 95, 7037 (2004).

12. A.V. Goncharov, A.A. Zhukov, M.A. Ghanem, P.N. Bartlett, R. Boardman, H. Fangohr, and P.A.J. de Groot, JMMM, in press.

13. A.A. Zhukov, E.T. Filby, M.A. Ghanem, P.N. Bartlett and P.A.J. de Groot, Physica C 404455 (2004).

14. F. E. Luborsky, J. Appl. Phys. 32 171S (1961).

15. E.F.Kneller, F. E. Luborsky, J. Appl. Phys. 34656 (1963).

16. R.P.Cowburn, JMMM 242-245, 505 (2002). K.Y. Guslienko and K.L. Metlov, Phys.Rev.B 63, 100403 (2001).

17. P. Vavassori, O.Donzelli, V. Metlushko. M. Grimsditch, B.Ilic, P.Neuzil and R.Kumar, J.Appl.Phys. 88, 999 (2000).

18. The "Object Oriented Micromagnetic Framework" developed at NIST, Gaithersburg - http://math.nist.gov/oommf/.

19. K. Maki, Physics 1, 127 (1964).

20. R. Skomski and J.M.D. Coey, Permanent magnetism (Institute of Physics Publishing, 1999).

21. R.C. O'Handley, Modern magnetic materials, (Wiley, 2000).

22. A.A.Zhukov et al., will be published.

23. E.A.Lynton, Superconductivity (Meuthuen, 1969)

24. V.V. Moshchalkov, L. Gielen, C. Strunk, R. Jonckheere, X. Qiu, C. Vanhaesendonck and and Y. Bruynseraede, Nature, 373, 319 (1995).

25. V.V. Moshchalkov, M. Baert, V.V. Metlushko, E. Rosseel, M.J. Van Bael, K. Temst, R. Jonckheere and Y. Bruynseraede, Phys.Rev.B, 54, 7385 (1996).

26. A.A. Zhukov et al., will be published

27. G.S. Mkrtchyan and V.V. Schmidt, JETP 34, 37 (1972).

28. D.R. Nelson and V.M. Vinokur, Phys.Rev.B 48, 13060 (1993). 